\title{
Hypotension Prediction Index: from proof-of-concept to proof-of-feasibility
}

\author{
llonka N. de Keijzer ${ }^{1}$. Jaap Jan $\operatorname{Vos}^{1} \cdot$ Thomas W. L. Scheeren $^{1}$
}

Received: 16 January 2020 / Accepted: 20 January 2020 / Published online: 23 January 2020

(c) Springer Nature B.V. 2020

Intraoperative hypotension $(\mathrm{IOH})$ is increasingly recognized as a major contributing factor associated with the development of postoperative complications in terms of renal [1-6], myocardial [6-8] and possibly, cerebral injury [9-11], despite substantial variability in literature regarding its exact definition $[12,13]$. As IOH is not only associated with perioperative morbidity but with perioperative mortality [5, 14-17] as well-which is the 3rd greatest global contributor to deaths after ischemic heart disease and stroke [18] — efforts should be made to reduce both the incidence and duration of $\mathrm{IOH}$. Hence, recently a consensus statement by the Perioperative Quality Initiative-3 workgroup [19] advises that for adults undergoing non-cardiac surgery, there is substantial evidence supporting that mean arterial pressure (MAP) should be kept above $60-70 \mathrm{mmHg}$ in order to reduce postoperative myocardial and renal injury, and death. Given that even brief periods of IOH may be harmful-e.g. after induction of anesthesia and before surgical incision [1] - it may be beneficial to change our current practice from a reactive approach (by monitoring the patient's actual hemodynamic status) $[20,21]$ to a proactive approach, by predicting vital signs [22], especially since (cumulatively) the longer a patients "spends" in $\mathrm{IOH}$, the more likely it is that this will adversely affect outcome [14]. The current advances in medical technology include the use of machinelearning based algorithms [23, 24] to analyze large datasets in order to provide clinically useful information. Such predictive analytics may help in substantiating such a proactive approach.

\section{In a nutshell: machine-learning algorithm in (bio)medical research}

Arthur L. Samuels was in 1959 the first one to describe the concept of machine-learning. It was described as "the programming of a computer to behave in a way which, if done by human beings or animals, would be described as involving the process of learning". The first machines were programmed to play checkers and in just a few hours the machines learned to play checkers better than the persons who programmed the computers [25]. Machine-learning algorithms differ substantially from traditional, rule-based algorithms. In such traditional algorithms, a pre-defined situation is handled by pre-defined criteria as set by the programmer. While this may be accurate, e.g. the administration of anesthetics using target-controlled infusion algorithms [23], its performance depends on the exact definitions and criteria, as set by the programmer. In machine learning, multiple input variables (features) are associated with output variable(s). Different forms of machine-learning exist, yet conceptually in medical practice, it allows the analysis of large (patient-derived) datasets for given output variables, so that a predictive model can be constructed in which the structure and weight of all identified features are considered by the algorithm. In the past decade, the development and use of machine-learning algorithms has grown exponentially in many sectors, including medical care. Recently, guidelines were developed for developing machine-learning algorithms and for reporting in (bio)medical journals [26].

\subsection{Development and validation of the Hypotension Prediction Index (HPI)}

A good example of a machine-learning derived algorithm that may be used perioperatively to improve patient outcome, is the commercially available HPI algorithm (Edwards Lifesciences, Irvine, USA) [27]. The HPI variable givesin a unitless range between 0 and 100-the likelihood of
Thomas W. L. Scheeren

t.w.1.scheeren@umcg.nl

1 Department of Anesthesiology, University Medical Center Groningen, University of Groningen, Hanzeplein 1, 9700 RB Groningen, The Netherlands 
impending hypotension-i.e. MAP $<65 \mathrm{mmHg}$-in the next 5-15 min, while the patient is still hemodynamically stable. The HPI algorithm continuously analyzes the arterial pressure waveform and has been trained on a large dataset from over 1300 patients (both perioperatively and from ICU patients) that contained many hypotensive episodes $(\mathrm{n}=25,461)$ [27].

The HPI algorithm works on top of the FloTrac algorithm. The latter allows the identification of different pulse waves, and splits data into frames of $20 \mathrm{~s}$ for subsequent analyses. HPI splits the individual pulse waves in distinct (physiological) phases, and a total of 3022 individual characteristics of the waveform(s) are analyzed, as well as their interrelation. Based on the "training" of the HPI algorithm, the likelihood of impending hypotension is given. Further details on the development of HPI are given elsewhere [21, 27, 28].

After internal validation, the derived model was prospectively externally validated in a cohort of 204 surgical (perioperative) patients, containing 1923 episodes of hypotension. Here, hypotension could be predicted $15 \mathrm{~min}$ in advance with a specificity of $83 \%$ and a sensitivity of $84 \%$ with an area under the curve (AUC) of 0.91, and up to $5 \mathrm{~min}$ in advance with a sensitivity $87 \%$, specificity of $88 \%$ and AUC of 0.95 [27].

Until recently, two studies [28, 29] have evaluated the performance of HPI in predicting hypotension. In one of these studies in 255 patients undergoing major surgery [28] HPI and other commonly used hemodynamic variables were analyzed in order to predict hypotension. HPI was superior in predicting hypotension compared to mean arterial pressure itself, the change in mean arterial pressure over $3 \mathrm{~min}$, and any other hemodynamic variable such as stroke volume variation, cardiac output, heart rate, shock index and pulse pressure. HPI could predict hypotension $5 \mathrm{~min}$ in advance with a sensitivity and specificity of $86 \%$ with an AUC of 0.93 .

In another study [29] in 23 patients undergoing (cardio) vascular surgery, the discrimination and calibration levels of the HPI 5-7 min before occurrence of a hypotensive event were evaluated. The HPI had a poor level of calibration at the cut-off value of 85 , as sensitivity was only $62 \%$ and specificity was $77 \%$, with an associated negative predictive value (NPV) of $98 \%$ and a positive predictive value (PPV) of only $13 \%$. When the cut-off value was increased to a HPI value of 98 , PPV increased to $64 \%$ and NPV decreased to $95 \%$.

\subsection{Implementation of HPI in protocolized perioperative hemodynamic care}

In this issue of the journal, Schneck et al. [30] provide the first true clinical (feasibility) study that evaluates the value of HPI in minimizing the incidence and duration of $\mathrm{IOH}$, in addition to the use of protocolized perioperative hemodynamic care. In this study, 90 patients undergoing total hip arthroplasty under general anesthesia were included. An intervention group $(\mathrm{n}=25)$ using goaldirected therapy guided by HPI was compared to a prospective control group $(n=24)$. In the intervention group, a HPI value $>80$ triggered either colloid administration (if stroke volume variation (SVV) $>12 \%$ ), or the administration of inotropes [if Cardiac Index (CI) was under the individual threshold], or the administration of vasopressors (when CI was above the individual threshold). In the control group, it was aimed to keep MAP $>65 \mathrm{mmHg}$, without using HPI. Both these prospective groups were additionally compared with a historical group $(n=50)$, in whom 'routine' anesthetic management was performed according to institutional practice (and in whom no study monitoring was applied). Interestingly, the incidence of hypotension was eliminated in the intervention group: with a median absolute time spent at a $\mathrm{MAP} \leq 65 \mathrm{mmHg}$ of $0 \mathrm{~s}$, albeit with a range of $0-140 \mathrm{~s}$. In comparison: in the prospective control group, the median hypotension time was 640 [195-1315] seconds, which was similar to that in the historical control group (660 [180-1440] seconds). Although one may consider this study only as a first-step in the clinical implementation of machine-learning algorithms (i.e. HPI) in goal-directed therapy protocols, it is a very important first step, as it clearly shows that it may allow reducing the incidence and duration of $\mathrm{IOH}$ to almost zero in most patients treated using HPI monitoring. Furthermore, it is intriguing that in the prospective control group, in which it was aimed to keep MAP above $65 \mathrm{mmHg}$ by the treating clinicians who were aware of the fact that they actively participated in a study that focused on the reduction of $\mathrm{IOH}$, hypotension did still occurwith a duration that was equivalent to the historical control group in which 'only' institutional practice was performed. This observation emphasizes that a reactive treatment of $\mathrm{IOH}$ may indeed be inferior to a proactive treatment of impending $\mathrm{IOH}$ in reducing its incidence and duration.

Despite the promising results of this study in reducing $\mathrm{IOH}-$ that obviate(s) the need for future studies to assess the effect of HPI-based treatment on actual patient outcomesome remarks should be made.

Firstly, the current study has a very limited external validity since it is only a single-center study in patients undergoing one type of surgical procedure, both of which may be subject to substantial bias with respect to the primary outcome variable, in this case: IOH. It remains to be decided whether treatment based on HPI provides equivalent reductions in $\mathrm{IOH}$ in other patient populations in whom the risks of postoperative morbidity and mortality are actually increased based on their co-morbidity or the surgical procedure. Also, the study was aimed to show a reduction in the time of IOH only, and as such, does not provide any 
evidence of a beneficial impact on patient outcome in terms of postoperative morbidity and mortality whatsoever.

Secondly, the results of the study do not provide sufficient information to elucidate what exactly decreased the incidence of $\mathrm{IOH}$ in the intervention group. Was it the (reduced) amount of fluids - or the timing of its administration? Was it the correct indication of vasopressors and/or inotropes, at the right moment? The results of the study would have been more clear if information were provided on goal-directed fluid therapy related variables, i.e. SVV and CI, as it is now not possible to discriminate whether it was mainly HPI "itself", or the goal-directed fluid therapy in which HPI was embedded, that resulted in the reduction of IOH. In an ideal setting, another control group was defined in which HPI was monitored-but blinded - and goal-directed fluid therapy was performed. Most likely, it may have been a combination of the factors mentioned above, that reduced the incidence of IOH. In addition, the "secondary screen" variables that come along with the HPI algorithm and are meant to help deciding whether or not to treat any of the underlying mechanisms that may cause hypotension (i.e. dynamic preload variables for preload, $\mathrm{dP} / \mathrm{dt}_{\max }$ for contractility and dynamic arterial elastance for afterload; details are explained elsewhere [21]), were not used by the authors, which would otherwise have been very relevant as it would have allowed an initial assessment whether these variables truly help in optimizing hemodynamics.

Another important aspect, often neglected in hemodynamic studies, is the influence and depth of general anesthesia. Cumulatively, the authors found slight, but statistically significant differences in the dosage of fentanyl and sevoflurane between the prospective groups. It may well be that the "trick" in reducing IOH lies in optimizing preload by timely administrating the right amount of fluids, and in optimizing contractility and afterload by the timely and right dosage of inotropes and vasopressors, respectively, while adjusting the level of general anesthesia in order to maintain hemodynamic stability — even together with the information provided by the "secondary screen" variables. Such complex processes are however not easily recognized in studies as performed by Schneck et al. While they were unable to retrieve BIS and MAC levels-so that a comparable depth of anesthesia could not be proven - it may have been true that in the control group(s), there was a deeper level of general anesthesia, which may have resulted in longer periods of $\mathrm{IOH}$.

Finally, it is unclear when exactly IOH occurred, and what the temporal distribution of $\mathrm{IOH}$ was; were most of the hypotensive events occurring after induction of general anesthesia (but before surgical incision) or did IOH mainly occur intraoperatively, after the surgeon had started the procedure? Such information may provide important information on the conduct and influence of the induction of general anesthesia, since it was demonstrated previously that even post-induction hypotension contributes to the development of postoperative morbidity [1].

\section{Conclusion}

Despite these critical remarks, this elegant, very first proofof-feasibility study on the use of HPI in the perioperative setting provides substantial evidence that a HPI-triggered goal-directed therapy approach may reduce the incidence and duration of $\mathrm{IOH}$, which is a well-recognized factor that is associated with the development of postoperative morbidity and mortality. It may just be one of the very first studies in this new decade that helps improving perioperative patient safety further by using innovative technology such as the commercially available Hypotension Prediction Index.

\section{Compliance with ethical standards}

Conflict of interest TWLS received research grants and honoraria from Edwards Lifesciences (Irvine, CA, USA) and Masimo Inc. (Irvine, CA, USA) for consulting and lecturing and from Pulsion Medical Systems SE (Feldkirchen, Germany) for lecturing. TWLS is associate editor of the Journal of Clinical Monitoring and Computing but had no role in the handling of this paper. JJV and IDK have no conflicts of interest to declare.

\section{References}

1. Maheshwari K, Turan A, Mao G, Yang D, Niazi AK, Agarwal D, Sessler DI, Kurz A. The association of hypotension during noncardiac surgery, before and after skin incision, with postoperative acute kidney injury: a retrospective cohort analysis. Anaesthesia. 2018;73:1223-8.

2. Walsh M, Devereaux PJ, Garg AX, Kurz A, Turan A, Rodseth RN, Cywinski J, Thabane L, Sessler DI. Relationship between intraoperative mean arterial pressure and clinical outcomes after noncardiac surgery: toward an empirical definition of hypotension. Anesthesiology. 2013;119:507-15.

3. Salmasi V, Maheshwari K, Yang D, Mascha EJ, Singh A, Sessler DI, Kurz A. Relationship between intraoperative hypotension, defined by either reduction from baseline or absolute thresholds, and acute kidney and myocardial injury after noncardiac surgery: a retrospective cohort analysis. Anesthesiology. 2017;126:47-65.

4. Sun LY, Wijeysundera DN, Tait GA, Beattie WS. Association of intraoperative hypotension with acute kidney injury after elective noncardiac surgery. Anesthesiology. 2015;123:515-23.

5. Khanna AK, Maheshwari K, Mao G, Liu L, Perez-Protto SE, Chodavarapu P, Schacham YN, Sessler DI. Association between mean arterial pressure and acute kidney injury and a composite of myocardial injury and mortality in postoperative critically ill patients: a retrospective cohort analysis. Crit Care Med. 2019;47:910-7.

6. Mathis MR, Naik BI, Freundlich RE, Shanks AM, Heung M, Kim M, Burns ML, Colquhoun DA, Rangrass G, Janda A, Engoren MC, Saager L, Tremper KK, Kheterpal S. Preoperative risk and the association between hypotension and postoperative acute 
kidney injury. Anesthesiology. 2020. https://doi.org/10.1097/ ALN.0000000000003063.

7. Hallqvist L, Martensson J, Granath F, Sahlen A, Bell M. Intraoperative hypotension is associated with myocardial damage in noncardiac surgery: an observational study. Eur J Anaesthesiol. 2016;33:450-6.

8. Sessler DI, Khanna AK. Perioperative myocardial injury and the contribution of hypotension. Intensive Care Med. 2018;44:811-22.

9. Nagre AS. Perioperative stroke-prediction, prevention, and protection. Indian J Anaesth. 2018;62:738-42.

10. Bijker JB, Persoon S, Peelen LM, Moons KG, Kalkman CJ, Kappelle LJ, van Klei WA. Intraoperative hypotension and perioperative ischemic stroke after general surgery: a nested case-control study. Anesthesiology. 2012;116:658-64.

11. Sun LY, Chung AM, Farkouh ME, van Diepen S, Weinberger J, Bourke M, Ruel M. Defining an intraoperative hypotension threshold in association with stroke in cardiac surgery. Anesthesiology. 2018;129:440-7.

12. Bijker JB, van Klei WA, Kappen TH, van Wolfswinkel L, Moons $\mathrm{KG}$, Kalkman CJ. Incidence of intraoperative hypotension as a function of the chosen definition: literature definitions applied to a retrospective cohort using automated data collection. Anesthesiology. 2007;107:213-20.

13. Wesselink EM, Kappen TH, Torn HM, Slooter AJC, van Klei WA. Intraoperative hypotension and the risk of postoperative adverse outcomes: a systematic review. Br J Anaesth. 2018;121:706-21.

14. Monk TG, Bronsert MR, Henderson WG, Mangione MP, SumPing ST, Bentt DR, Nguyen JD, Richman JS, Meguid RA, Hammermeister KE. Association between intraoperative hypotension and hypertension and 30-day postoperative mortality in noncardiac surgery. Anesthesiology. 2015;123:307-19.

15. Mascha EJ, Yang D, Weiss S, Sessler DI. Intraoperative mean arterial pressure variability and 30-day mortality in patients having noncardiac surgery. Anesthesiology. 2015;123:79-91.

16. Stapelfeldt W, Yuan H, Dryden J, Strehl K, Cywinski J, Ehrenfeld J, Bromley P. The SLUScore: a novel method for detecting hazardous hypotension in adult patients undergoing noncardiac surgical procedures. Anesth Analg. 2017;124:1135-52.

17. Vincent J, Nielsen ND, Shapiro NI, Gerbasi ME, Grossman A, Doroff R, Zeng F, Young PJ, Russell JA. Mean arterial pressure and mortality in patients with distributive shock: a retrospective analysis of the MIMIC-III database. Ann Intensive Care. 2018;8:107.

18. Nepogodiev D, Martin J, Biccard B, Makupe A, Bhangu A. Global burden of postoperative death. Lancet. 2019;393:401.

19. Sessler DI, Bloomstone JA, Aronson S, Berry C, Gan TJ, Kellum JA, Plumb J, Mythen MG, Grocott MPW, Edwards MR, Miller TE, Perioperative Quality Initiative-3 Workgroup, POQI Chairs, Miller TE, Mythen MG, Grocott MP, Edwards MR, Physiology
Group, Preoperative Blood Pressure Group, Intraoperative Blood Pressure Group, Postoperative Blood Pressure Group. Perioperative quality initiative consensus statement on intraoperative blood pressure, risk and outcomes for elective surgery. $\mathrm{Br} \mathrm{J}$ Anaesth. 2019;122:563-74.

20. Saugel B, Kouz K, Hoppe P, Maheshwari K, Scheeren TWL. Predicting hypotension in perioperative and intensive care medicine. Best Pract Res Clin Anaesthesiol. 2019;33:189-97.

21. Vos JJ, Scheeren TWL. Intraoperative hypotension and its prediction. Indian J Anaesth. 2019;63:877-85.

22. Vistisen ST, Johnson AEW, Scheeren TWL. Predicting vital sign deterioration with artificial intelligence or machine learning. J Clin Monit Comput. 2019;33:949-51.

23. Connor CW. Artificial intelligence and machine learning in anesthesiology. Anesthesiology. 2019;131:1346-59.

24. Rajkomar A, Dean J, Kohane I. Machine learning in medicine. N Engl J Med. 2019;380:1347-58.

25. Samuel AL. Some studies in machine learning using the game of checkers. IBM J Res Dev. 1959;3:210-29.

26. Luo W, Phung D, Tran T, Gupta S, Rana S, Karmakar C, Shilton A, Yearwood J, Dimitrova N, Ho TB, Venkatesh S, Berk M. Guidelines for developing and reporting machine learning predictive models in biomedical research: a multidisciplinary view. $\mathrm{J}$ Med Internet Res. 2016;18:e323.

27. Hatib F, Jian Z, Buddi S, Lee C, Settels J, Sibert K, Rinehart J, Cannesson M. Machine-learning algorithm to predict hypotension based on high-fidelity arterial pressure waveform analysis. Anesthesiology. 2018;129:663-74.

28. Davies SJ, Vistisen ST, Jian Z, Hatib F, Scheeren TWL. Ability of an arterial waveform analysis-derived hypotension prediction index to predict future hypotensive events in surgical patients. Anesth Analg. 2020;130:352-9.

29. Ranucci M, Barile L, Ambrogi F, Pistuddi V, Surgical, and Clinical Outcome Research (SCORE) Group. Discrimination and calibration properties of the hypotension probability indicator during cardiac and vascular surgery. Minerva Anestesiol. 2019;85:724-30.

30. Schneck E, Schulte D, Habig L, Ruhrmann S, Edinger F, Markmann M, Habicher M, Rickert M, Koch C, Sander M. Hypotension prediction index based protocolized haemodynamic management reduces the incidence and duration of intraoperative hypotension in primary total hip arthroplasty: a single centre feasibility randomised blinded prospective interventional trial. J Clin Monit Comput. 2019. https://doi.org/10.1007/s10877-019-00433-6.

Publisher's Note Springer Nature remains neutral with regard to jurisdictional claims in published maps and institutional affiliations. 